\title{
Die Freizügigkeit und die Realisierung der Regelungen im Bereich des Sozialschutzes zwischen Polen und Deutschland aus der Sicht der Sozialversicherungsanstalt/Abteilung in Oppeln
}

\section{Bogdan Borecki}

A. Einführung und Entwicklung $\quad 257$

$\begin{array}{ll}\text { I. Entwicklung bis } 1990 & 257\end{array}$

II. Statistischer Hintergrund 259

III. Abschluss des neuen Sozialversicherungsabkommens 259

IV. Beitritt Polens zur EU 260

B. Anwendungsfragen $\quad 261$

I. Anwendbarkeit der rechtlichen Grundlagen 261

II. Folgen einer Rückkehr nach Polen 262

III. Weitere Fragen 263

1. Leistungsbewilligung $\quad 263$

2. Leistungsexport 264

3. Verschiedene Anspruchsvoraussetzungen 265

4. Sachleistungen 266

$\begin{array}{ll}\text { C. Schluss } & 266\end{array}$

\section{A. Einführung und Entwicklung}

\section{Entwicklung bis 1990}

Dieser Bericht ist ein Versuch, einen kleinen Ausschnitt der internationalen Zusammenarbeit im Bereich des Sozialschutzes darzustellen. Er betrifft die Aufgaben, die durch die Abteilung für Abwicklung internationaler Verträge der Sozialversicherungsanstalt in Oppeln im Rahmen der deutsch-polnischen Zusammenarbeit umgesetzt werden.

Eine der ersten Bestimmungen in diesem Bereich ist das Abkommen zwischen der Volksrepublik Polen und der DDR vom 13. Juli 1957 über die Zusammenarbeit auf dem Gebiet der Sozialpolitik. Das ist ein bilaterales Abkommen mit einem „territorialen“ Charakter. Auf der polnischen Seite betrifft es Arbeiter, die in Polen wohnen und in der Vergangenheit in der DDR gearbeitet haben. Der territoriale Charakter besteht darin, 
dass alle Beschäftigungszeiten, die in der ehemaligen DDR zurückgelegt wurden und für die Sozialversicherungsbeiträge in der DDR abgeführt wurden, durch den polnischen Rententräger, die Sozialversicherungsanstalt, so angerechnet werden, als wären diese Zeiten auf polnischem Gebiet zurückgelegt worden, umd zwar sowohl im Hinblick auf den Leistungsanspruch als auch im Hinblick auf die Leistungshöhe. Dieses Abkommen ist weiterhin aktuell und betrifft alle Versicherungszeiten, die bis zum 2 . Oktober 1990, dem letzten Tag vor dem Vereinigungsabkommen zwischen der DDR und der BRD, auf dem Gebiet der ehemaligen DDR zurückgelegt wurden.

Als nächster Schritt der deutsch-polnischen Zusammenarbeit, die durch die Sozialversicherungsanstalt in Oppeln realisiert wird, ist das nicht veröffentlichte Abkommen vom 20.07.1967 zu erwähnen. Dieses Abkommen wurde zwischen der Sozialversicherungsanstalt, dem Auslandsrentenbüro in Warschau und den Versorgungsämtern in Ravensburg, Münster, Gelsenkirchen und Hamburg geschlossen; es betrifft die Zahlung von Leistungen an Invaliden der ehemaligen Wehrmacht und ihre Familien sowie ihnen gleich gestellte Personen. In den Jahren 1968-1989 wurden von der Zweigstelle Oppeln im Auftrag des Auslandsrentenbüros Versorgungsleistungen an 23890 Berechtigte ausgezahlt.

Ferner hat die Abteilung in Oppeln, ähnlich allen anderen in diesen Jahren agierenden Abteilungen der Sozialversicherungsanstalt, die Bestimmungen des deutschpolnischen Abkommens vom 09.10.1975 im Bereich der Renten- und Unfallversicherung welches zum 01.05.1976 in Kraft getreten ist, schon ab 1977 angewendet. Eine Vereinbarung des Ministeriums für Arbeit, Löhne und soziale Angelegenheiten der Volksrepublik Polen und des Bundesministeriums für Arbeit und Sozialordnung der BRD hinsichtlich der Realisierung des Abkommens wurde am 11.01.1977 in Warschau unterschrieben, am 20.09.1977 wurde in Berlin eine Verwaltungsvereinbarung der Verbindungsstellen für Rentenversicherungen unterzeichnet und am 26.10.1977 wurde in Bonn eine Administrationsvereinbarung der Verbindungsstellen für Unfallversicherungen getroffen.

Die steigende Migration der Bevölkerung von Oppelner Schlesiern in die Bundesrepublik Deutschland führte zur Gründung einer selbständigen Abteilung für die Abwicklung internationaler Verträge zwischen der Volksrepublik Polen und der BRD auf der Grundlage des Beschlusses des Vorsitzenden der Sozialversicherungsanstalt vom 02.03.1989 in der Zweigstelle der Sozialversicherungsanstalt in Oppeln . Der laufende Wandel im sogenannten Ostblock bewirkte eine gewaltige Migrationswelle der DDRBürger in die BRD im Sommer 1989 und zahlreiche Demonstrationen der DDRBevölkerung im Hinblick auf die deutsche Einheit. Dies übte einen immer größeren Druck auf die deutschen Behörden aus. Am 09.11.1989 wurden die Grenzen zwischen der DDR und der BRD geöffnet und knapp ein Jahr später, am 03.10.1990, kam es zur Vereinigung der beiden deutschen Staaten auf der Grundlage des Vereinigungsabkommens vom 31.08.1990. 


\section{Statistischer Hintergrund}

Die Zahl der Bevölkerung der Oppelner Woiwodschaft übersteigt aktuell eine Million Einwohner. Bei einer so kleinen Population betrug die Auslandsmigration (auf Dauer) - hauptsächlich nach Deutschland - 237093 Personen in den Jahren 1951-2009. Davon sind in den Jahren 1951-1990 (die durch das Abkommen vom 9.10.1975 erfasst wurden) 174208 Personen ausgewandert, 62885 Personen in den Jahren 1991-2009 und nach dem Beitritt Polens zur Europäischen Union, also in den Jahren 2004-2009, 22322 Personen.

Unabhängig von der Emigration auf Dauer gibt es auch eine Emigration auf Zeit, die nach Untersuchungen des Hauptstatistikamtes im Jahr 2002 in der Oppelner Region 105000 Personen und im Jahr 2011 über 150000 Personen betrug. ${ }^{1}$

\section{Abschluss des neuen Sozialversicherungsabkommens}

Zwischen dem Ministerium für Arbeit und Sozialordnung der Bundesrepublik Deutschland und dem Arbeits- und Sozialpolitikministerium der Republik Polen wurden Gespräche hinsichtlich einer Änderung des bisherigen Abkommens über Renten- und Unfallversorgung aus dem Jahr 1975 aufgenommen, das auf dem Territorialprinzip basierte. In dieser Zeit wandelte sich Europa. Es erfolgte eine grundlegende politische Wende in den Staaten Mittel- und Osteuropas. In Polen fand ein sozialer und wirtschaftlicher Wandel statt. Es wurden Verhandlungen zur Vereinigung der ehemaligen DDR mit der BRD und zur Gründung eines gesamtdeutschen Staates aufgenommen. Diese Veränderungen trugen zu einer Neuregelung der deutsch-polnischen Verhältnisse hinsichtlich der sozialen Versorgung und zum Ersatz der territorialen Regel durch die Regel des Leistungsexports bei. Ein neues Abkommen wurde vorbereitet - das Sozialversicherungsabkommen über Soziale Sicherheit vom 08.12.1990.

Dieses Abkommen wurde im Dezember 1990 unterschrieben. In Polen wurde es am 25. September 1991 ratifiziert und trat am 1. Oktober 1991 in Kraft. Um das Abkommen praktisch realisieren zu können, mussten viele Vorkehrungen in prozessualem Sinne getroffen werden. Bereits am 3. Oktober 1991 wurde eine Vereinbarung von fünf deutschen Versicherungsanstalten und der polnischen Sozialversicherungsanstalt über die Ausführung des Abkommens im Rahmen der Rentenversicherung vereinbart und unterschrieben. Diese Einigung regelte fast alle Probleme hinsichtlich des Verfahrens und ermöglichte die Prüfung von Rentenanträgen unter Anwendung des neuen Abkommens.

1 Auf der Grundlage eigener Statistiken: Radzińskiego, Katedra Rynku Pracy i Kapitału Ludzkiego, Politechnika Opolska, 2009. 
Auf der Grundlage eines Schreibens des Vorsitzenden der Sozialversicherungsanstalt vom 11.07.1991, rief der Direktor der Zweigstelle in Oppeln mit der Verordnung Nr. 19 vom 24.07.1991 zum 01.08.1991 die Abteilung der deutsch-polnischen Renten ins Leben, die als einzige in Polen in Zusammenarbeit mit dem Auslandsrentenbüro in Warschau das Abkommen im Bereich der Unfallleistungen umsetzen sollte.

Die bedeutendsten Regelungen des Abkommens im Bereich der gesetzlichen Unfallund Rentenversicherung betrafen:

- Die Gleichbehandlung der polnischen und deutschen Staatsangehörigen, d.h., jeder Vertragsstaat hat die Staatsangehörigen des anderen Vertragstaates und die eigenen Staatsangehörigen rechtlich gleich zu behandeln, sofern sie in einem Vertragsstaat wohnen,

- die Berücksichtigung der in beiden Staaten zurückgelegten Arbeitszeiten hinsichtlich der Erfüllung der Voraussetzungen für die Gewährung polnischer und deutscher Rentenleistungen,

- die Berechnung der Höhe der polnischen und der deutschen Rentenleistungen und

- die weitere Anwendung des Abkommens zwischen der Volksrepublik Polen und der BRD über die Renten- und Unfallversorgung vom 09.10.1975.

Der sachliche Anwendungsbereich beider Abkommen erstreckte sich hauptsächlich auf die Gewährung von Rentenleistungen aus einer Arbeitnehmerversicherung. Das betrifft sowohl die Möglichkeit der Berücksichtigung der Arbeitszeiten, die in beiden Staaten zurückgelegt wurden, bei Bewilligung der Rentenleistung, als auch den Leistungsexport, der sich aus dem Abkommen vom 08.12.1990 ergibt. Es ist zu betonen, dass die Regelungen der Abkommen auch auf andere Versicherungszeiten als diejenigen in einer Arbeitnehmerversicherung angewendet werden konnten, z.B. auf die Verrichtung einer selbständigen, auch künstlerischen Tätigkeit. Diese Zeiten mussten bei der Feststellung der Arbeitsleistungen berücksichtigt worden sein und als letzte Versicherungszeit musste im Versicherungsverlauf eine Arbeitnehmerversicherung aufgeführt sein. Im Falle der Nichterfüllung dieser Voraussetzungen wurde die Leistung anhand der innerstaatlichen Vorschriften jedes der Vertragsstaaten ohne Berücksichtigung des deutsch-polnischen Abkommens von 1975 oder von 1990 errechnet.

\section{Beitritt Polens zur EU}

Ein besonderes Augenmerk wird auf den sachlichen Anwendungsbereich der beiden bilateralen Abkommen gerichtet, weil er im Vergleich zum sachlichen Anwendungsbereich der Europäischen Verordnungen zur Koordinierung der Sozialversorgungssysteme (EWG) 1408/71 und 574/72 sehr eingeschränkt ist. Dieser Unterschied im sachlichen Anwendungsbereich der bilateralen Abkommen zeigte sich auch im Umfang der Aufgaben, die die Sozialversicherungsanstalt in Oppeln bei deren Durchführung wahrnahm. 
Durch den Beitritt Polens zur Europäischen Union trat Polen auch im Bereich der sozialen Sicherheit in eine gemeinsame rechtliche Ordnung ein. Ab dem 01.05.2004 wurden die deutsch-polnischen Verhältnisse im Bereich der sozialen Sicherheit durch die folgenden Vorschriften geregelt:

- Verordnung des Rates (EWG) Nr. 1408/71 vom 14.06.1971 über die Anwendung der Systeme der sozialen Sicherheit auf Arbeitnehmer und Selbständige sowie deren Familienangehörige, die innerhalb der Gemeinschaft zu- und abwandern ab dem 01.05.2010 ersetzt durch die Verordnung des Europäischen Parlamentes und des Rates (EG) Nr. 883/2004 vom 29.04.2004 zur Koordinierung der Systeme der sozialen Sicherheit,

- Verordnung Nr. 574/72 vom 21.03.1972 über die Durchführung der Verordnung (EWG) Nr. 1408/71, die am 01.05.2010 durch die Verordnung des Europäischen Parlamentes und des Rates (EG) Nr. 987/2009 vom 16.09.2009 zur Festlegung der Modalitäten für die Durchführung der Verordnung (EG) Nr. 883/2004 ersetzt wurde und

- deutsch-polnisches Abkommen vom 09.10.1975 (territoriales Abkommen).

\section{B. Anwendungsfragen}

\section{Anwendbarkeit der rechtlichen Grundlagen}

Ähnlich dem Abkommen vom 08.12.1990 zwischen Polen und Deutschland, regeln die oben genannten Verordnungen, dass die entsprechenden Anstalten jedes Mitgliedsstaates der Union einen Rentenleistungsanspruch - gemäß der innerstaatlichen Gesetzgebung - unter Berücksichtigung der Versicherungszeiten, die in anderen Mitgliedsstaaten zurückgelegt wurden, bestimmen und dass für die Berechnung der Leistungshöhe die Versicherungszeiten, die im entsprechenden Staat zurückgelegt wurden, in Verhältnis zu allen zurückgelegten Versicherungszeiten gesetzt werden müssen (,,pro rata temporis“-Regel). Das bedeutet, wieder ähnlich dem bilateralem Abkommen vom 08.12.1990, dass die Institutionen jedes der Staaten lediglich verpflichtet sind, die Kosten für Versicherungszeiten zu tragen, die auf dem eigenen Territorium zurückgelegt wurden (proportionales Abkommen).

Jedoch findet die oben genannte Regel keine Anwendung auf Personen, die ihren Wohnsitz bis zum 01.01.1991 von Polen nach Deutschland verlegt haben (oder umgekehrt) und bis zu diesem Datum Versicherungszeiten in Polen und/oder in Deutschland zurückgelegt haben. Auf diese Personen wurde das Abkommen vom 09.10.1975 angewendet. Durch die Übernahme dieses Abkommens in die gemeinschaftsrechtlichen Verordnungen (es wurde in den Anhang III der Verordnung Nr. 1408/71, dann in den Anhang II der Verordnung Nr. 883/2004 aufgenommen) sind die Regeln dieses Ab- 
kommens auf diese Personen weiterhin anzuwenden. Das bedeutet, dass die Rentenleistungen nach den innerstaatlichen Vorschriften von den Versicherungsanstalten des Vertragsstaates, in dem die berechtigte Person ihren Wohnsitz hat, gewährt und ausgezahlt werden, wobei Versicherungszeiten, die in dem zweiten Staat zurückgelegt wurden, anerkannt werden, als ob sie auf dem eigenen Territorium zurückgelegt worden seien, so dass der Staat des Wohnsitzes die finanziellen Folgen der Anerkennung dieser Zeiten trägt. Alle Forderungen und Ansprüche, die aus dem Abkommen vom 09.10.1975 hervorgehen, erlöschen also so lange nicht, wie die berechtigte Person ihren Wohnsitz nicht wechselt. Eine Verlegung des Wohnsitzes nach dem 31.12.1990 ins Territorium des zweiten Vertragsstaates oder eines Drittstaates bewirkt eine erneute Feststellung der Rentenleistung, jedoch jetzt in beiden Staaten: in Polen und in Deutschland. Daraus kann eine Feststellung der Rentenleistung in jedem der Staaten auf der Grundlage nur der Versicherungszeiten in diesem Staat in voller Höhe resultieren, oder auf der Grundlage der gemeinsamen Versicherungszeiten in beiden Staaten in Teilhöhe, die dem Anteil der Zeiten in einem Staat im Verhältnis zur Gesamtversicherungszeit entspricht.

\section{Folgen einer Rückkehr nach Polen}

Dieses Detail muss man insbesondere deshalb besonders betonen, weil viele Menschen denken, der Beitritt Polens zur Europäischen Union bedeute, man könne sich frei bewegen und eine schon bewilligte Leistung in dem Staat des aktuellen Wohnsitzes werde als Leistungsexport weiter gezahlt. Daher ist zu beobachten, dass eine große Zahl von Menschen unter Bewahrung des Leistungsanspruches, den die deutsche Anstalt aufgrund der in Polen zurückgelegten Arbeitszeiten bewilligt hat, nach Polen zurückkehren möchte. Sie treffen die Entscheidung zu ihrer Rückkehr, ohne sich darüber im Klaren zu sein, dass dies Einfluss auf den rechtlichen Status hat - nämlich die endgültige Nichtanwendung des Abkommens vom 09.10.1975. Diesen Menschen muss man bewusst machen, dass aufgrund der Wohnsitzverlegung auf der deutschen Seite die Rentenansprüche überprüft werden und die Leistung nach der polnischen Gesetzgebung festgestellt wird. Das ist keine einfache Angelegenheit, denn um einen polnischen Leistungsanspruch festzustellen, müssen die entsprechenden Dokumente vorgelegt werden, die die Bearbeitung des Rentenantrags ermöglichen. Die Betroffenen haben jedoch große Probleme mit der Vorlage dieser Dokumente. Sie waren sich nicht bewusst, dass sie die Dokumente aus den 60er oder 70er Jahren noch einmal benötigen würden. Sie waren bereits Rentner und der Leistungsanspruch schien bereits festgestellt. Ihrer Ansicht nach war doch alles schon geklärt! Das sind emotionale und schwierige Angelegenheiten auch für uns; wir bemühen uns, den Menschen zu helfen, indem wir versuchen, etwaige Informationen von den deutschen Versicherungsträgern zu erhalten, was uns oftmals auch gelingt, weil die deutsche Seite Kopien von Dokumenten angefertigt und aufbewahrt hat. Doch nicht jede Angelegenheit findet ein gutes Ende und es kommt vor, 
dass wir den polnischen Leistungsanspruch ablehnen müssen, weil wir keine Dokumente besitzen, auf deren Grundlage wir einen Leistungsanspruch anerkennen könnten. Eine separate Gruppe bilden die Rentner, denen eine polnische Leistung bewilligt wurde, bevor sie vor dem 01.01.1991 Polen verlassen haben. Man kann sagen, dass diese Personen Glück hatten, weil sich in den ZUS-Archiven Dokumente befinden, die eine Neufeststellung der alten Leistung ermöglichen.

Ein Wechsel des Wohnortes bedeutet nicht nur die Notwendigkeit der Neufeststellung der Leistung oder deren Höhe durch die Sozialversicherungsanstalt, sondern auch den Bezug einer niedrigeren Leistung aufgrund der Differenz zwischen der Höhe der deutschen und der polnischen Leistung. Nicht selten ist noch ein Erstattungsanspruch der deutschen Versicherungsanstalt zu begleichen, der durch eine Überzahlung der deutschen Leistung nach der Rückkehr nach Polen entstanden ist.

\section{Weitere Fragen}

Nach Einleitung der Durchführung der Aufgaben, die mit der Einführung des Gemeinschaftsrechtes im Bereich der sozialen Sicherheit entstanden, kamen neue Aspekte der scheinbar bereits geregelten Aufgaben zum Vorschein. Grund dafür war der schon oben erwähnte, sehr weit gefasste sachliche und persönliche Anwendungsbereich der Europäischen Verordnungen.

\section{Leistungsbewilligung}

Zunächst ist die Leistungsbewilligung zu nennen; ab Geltung der Verordnungsvorschriften ist es möglich, die Leistung zu bewilligen und eine Exportleistung zu zahlen, nicht nur für Zeiten in einem Arbeitsverhältnis. Auch Personen, die Versicherungszeiten als Selbständige zurückgelegt haben sowie Kunstschaffende, Landwirte, Berufssoldaten, Bedienstete der Polizei, der Feuerwehr, des Strafvollzuges etc. können sich nicht nur um den Export der in Polen bewilligten Leistung in den jetzigen Wohnstaat bemühen, sondern auch um die Prüfung des Leistungsanspruches unter Anwendung des Koordinierungsgrundsatzes - insbesondere der Regel über die Summierung der gesamten Versicherungszeiten.

Die oben genannte Tatsache hat vor allem auf deutscher Seite eine breit angelegte Informationsaktion ausgelöst, die auf Personen ausgerichtet war, die in ihrem Versicherungsverlauf solche Nichtarbeitnehmerzeiten aufwiesen. Das betraf die Mehrheit der Personen, die dem Abkommen vom 09.10.1975 unterlagen; vor allem die, die zuletzt in Polen eine selbständige Tätigkeit ausgeübt hatten. Aufgrund dieser Zeiten ergab sich für diese Menschen die Möglichkeit der Prüfung des polnischen Leistungsanspruches. Zuvor musste aber die Welle der Anfragen, die bei uns sowohl in Form von Briefen als auch telefonisch eintrafen, bewältigt werden. Jede Veränderung erzeugt einen größeren 
Bedarf an Informationen. Zur Veranschaulichung sollen die folgenden Zahlen dienen: Im Jahr 2004 erteilte die Abteilung für Abwicklung internationaler Verträge 10391 telefonische Auskünfte und vermerkte den Eingang von 49946 Briefen, im Jahr 2005 wurden 15127 Telefonate und 81438 Briefe registriert. Das ergibt eine Steigerung um 45\% bei der Zahl der Telefonate und eine Steigerung um fast $65 \%$ bezüglich des Korrespondenzeingangs.

\section{Leistungsexport}

Darüber hinaus ist der Leistungsexport zu erwähnen. Diese Aufgabe existierte bereits, weil die Abteilung für Abwicklung internationaler Verträge auf der Grundlage des Abkommens vom 08.12.1990 einen Export der polnischen Leistungen für Personen, die in Deutschland wohnten, durchführte. Jedoch wurde dieser Leistungsexport von einer deutschen Versicherungsanstalt durchgeführt. Dagegen sehen die Regelungen der Verordnungen vor, dass die Leistungen unmittelbar an die berechtigten Personen, die in einem Mitgliedsstaat wohnen, ausgezahlt werden. Durch die Veränderung der Leistungszahlung mussten wir von allen Leistungsempfängern die Bankverbindungen von persönlichen, bei verschiedenen Banken in Deutschland geführten Konten erheben. Zusätzlich mussten diese Konten nicht nur im IBAN-Format bezeichnet werden, sondern es musste auch der SWIFT/BIC-Code angegeben werden. Das war eine enorme Herausforderung, welche die Bewältigung der Unzufriedenheit der Klienten erforderte, dennzeitweilige Zahlungsprobleme und die mangelhafte oder fehlende Angabe der Kontonummern verursachten die Zahlungseinstellung. Die Realisierung dieser Aufgabe war auch dank der Zusammenarbeit mit unseren Partnern aus Deutschland möglich, die uns halfen, die Angelegenheit positiv und schnell abzuschließen.

Die Leistungszahlung auf die persönlichen Bankkonten der Leistungsempfänger stellt bis heute eine Schwierigkeit dar. Das betrifft die Rücknahme von zu Unrecht ausgezahlten Leistungen, beispielsweise, wenn die berechtigte Person verstorben ist, wir noch keine Information darüber erhalten haben und die Leistung daher weiter auszahlen. In der Vergangenheit waren solche Situationen sehr selten - meistens wurde die deutsche Versicherungsanstalt, die bei der Auszahlung vermittelte, über den Tod der berechtigten Person informiert und überwies das zu Unrecht ausgezahlte Geld zurück an die Sozialversicherungsanstalt.

Als nächste Schwierigkeit bei der Realisierung der unmittelbaren Zahlungen ist der Kurswechsel zu nennen, der Bedeutung erlangt im Falle der Rückzahlung einer Leistung, die auf ein deutsches Konto überwiesen, dort aber nicht verbucht wurde - kurz gesagt: ein Betrag wird in Zloty überwiesen und ein anderer Betrag kommt zurück, obwohl es weiter dieselbe Leistung ist.

Vor demselben Dilemma stehen auch unsere Leistungsempfänger, die ihre polnische Leistung umgerechnet in Euro aufgrund des Kurswechsels jeden Monat in einer anderen Höhe bekommen. 
Das Ausmaß dieser Probleme wird erst dann klar, wenn man die Anzahl der Auszahlungen bedenkt. Bis zum Beitritt Polens zur Europäischen Union am 30.04.2004 zahlte unsere Abteilung Leistungen auf der Grundlage des deutsch-polnischen Abkommens von 1990 an 7679 Personen aus; im Dezember 2004 stieg diese Zahl auf 7993 Personen an, wobei als Grundlage der Auszahlungen hier auch die Verordnungen des EWG-Rates berücksichtigt wurden. Die erste Auszahlung unmittelbar an die Leistungsempfänger das heißt auf die persönlichen Konten der Begünstigten - erfolgte im Juli 2007; damals wurden Leistungen an 10623 Personen ausgezahlt; anderthalb Jahre später, im Dezember 2008, stieg diese Zahl auf 12377 Personen an und am 30.09.2011 betrug sie 16739 Personen.

\section{Verschiedene Anspruchsvoraussetzungen}

Eine weitere Ursache für Probleme sind die Unterschiede zwischen der deutschen und der polnischen Gesetzgebung. Die Vorschriften der Europäischen Union ersetzen die inländischen Systeme der sozialen Sicherheit nicht durch ein gemeinsames europäisches System. Aus politischer Sicht ist eine solche Angleichung auch nicht möglich, weil die Systeme der sozialen Sicherheit das Ergebnis mehrjähriger Traditionen darstellen, die tief in der nationalen Kultur verankert sind. Jeder Staat hat das Recht, alleine darüber zur entscheiden, wer versichert werden soll, nach welchen Vorschriften, welche Leistungen bewilligt werden und nach welchen Bedingungen, wie diese Leistungen angerechnet werden und welche Beitrage abgeführt werden sollen. Die Einführung der Koordinierung der Systeme der sozialen Sicherheit bezweckte nie, die Unterschiede zwischen den verschiedenen Inlandssystemen zu beseitigen und ein vereinheitlichtes, überstaatliches System zu erschaffen. Die Koordinierung akzeptiert die Unterschiede zwischen den verschiedenen Systemen und führt gleichzeitig rechtliche Instrumente ein, die eine Koexistenz der Systeme erlauben, wie zum Beispiel die Regeln der Gleichstellung, der Zusammenrechnung von Versicherungszeiten, von Arbeitszeiten oder von Wohnzeiten, sowie der Erhaltung der erworbenen Ansprüche. Die Koordinierung schafft aber nicht alle Probleme aus der Welt, wie zum Beispiel die Frage des Rentenalters. Nach polnischem Recht können Frauen im Alter von 55 Jahren und Männer im Alter von 60 Jahren die frühe Altersrente bekommen. Um den Anspruch für eine konkrete Person auf solch eine Leistung prüfen zu können, muss an die Sozialversicherungsanstalt ein Antrag mit allen nötigen Dokumenten gerichtet werden. Gemäß den EU-Vorschriften muss der Antrag in dem Staat des Wohnsitzes und nach den Regeln, die in diesem Staat herrschen, gestellt werden. Hier haben wir aber ein Problem beobachtet, das, wie wir hoffen, nun gelöst wurde und nicht wieder vorkommen wird. Die deutschen Versicherungsanstalten verweigerten nämlich die Annahme der Anträge von so ,jungen“ Personen; diese Personen wurden unmittelbar an die polnische Anstalt verwiesen. Dies war sehr unkomfortabel, nicht nur für die betreffenden Personen, weil sie nach Polen reisen mussten, sondern auch für uns. Wir hatten keine Informationen, we- 
der über den Aufenthalt des Antragstellers in Deutschland, seinen Versicherungsverlauf, ob er also weiterhin arbeitet und welche Versicherungszeiten er in Deutschland zurückgelegt hat, noch in Bezug auf die Versicherung im Bereich der Sachleistungen.

\section{Sachleistungen}

Hiermit kommen wir zum nächsten Thema, den Sachleistungen, also der Frage, in welchem Staat man krankenversichert ist. In Polen beschäftigt sich mit den Sachleistungen der Nationale Gesundheitsfonds. Die Sozialversicherungsanstalt arbeitet jedoch sehr eng mit diesem Fonds zusammen, weil sie als Leistungsträgerin verpflichtet ist, die Krankheitsversicherungsbeiträge für jeden Versicherten abzuziehen und an den Fonds weiterzuleiten. Man ziehe die folgende Situation in Betracht: ein 45-jähriger Mann, dem in Polen eine Rente wegen Erwerbsminderung bewilligt wurde, beschließt, seinen Wohnsitz nach Deutschland zu verlegen. Er stellt einen Antrag auf Leistungsexport der Rente nach Deutschland, legt alle Dokumente vor, woraufhin ein Bescheid ergeht und die Leistung ihm auf sein Konto in Deutschland überwiesen wird. Von dieser Rente werden aber weiterhin durch die Sozialversicherungsanstalt der Einkommensteuervorschuss und der Beitrag für die Krankenversicherung einbehalten und gleichzeitig wird der Nationale Gesundheitsfonds über den Wohnortwechsel des Rentners informiert. Wenn dieser Mann in Deutschland eine Arbeit aufnimmt - wozu er ein Recht hat, dann hat dies einen Einfluss auf den Vorrang der Sachleistungen; der Versicherte sollte wegen der verrichteten Arbeit und nicht wegen der Rentenbeziehung von der Krankenversicherung erfasst werden. Dennoch, auch wenn uns der Versicherte mitteilt, dass er in Deutschland arbeitet und die Einstellung der Einbehaltung des Krankenversicherungsbeitrags in Polen wünscht, kann die Sozialversicherungsanstalt nicht selbst darüber entscheiden - wir brauchen vielmehr einen Bescheid von dem Nationalen Gesundheitsfonds. Das einzige, was wir in dieser Angelegenheit tun können, ist, dem Versicherten mitzuteilen, wohin er sich mit seiner Bitte wenden muss, sowie die masowische Abteilung des Fonds über diese Situation zu informieren und deren Bescheid abzuwarten.

\section{Schluss}

Seit über 40 Jahren arbeiten wir mit den deutschen Sozialversicherungsanstalten zusammen. Die Geschichte der deutsch-polnischen Kontakte ist in sehr vielen Notizen, Berichten, Protokollen und Vereinbarungen über die Regelung der Probleme, die wir trotz unterschiedlicher Rechtssysteme und Unterschieden in der Organisation, der Administration der Sozialversicherung - gemeinsam regeln mussten, dokumentiert. Unsere Kontakte, die zuvor auf die Ausführung des Abkommens über die Sozialversicherung beschränkt waren, haben sich in den letzten Jahren auf andere Bereiche wie ärztliche 
Gutachten, Methoden der Beitragseinbehaltung und Organisation der Versicherungsanstalten ausgedehnt. Regelmäßig finden in Polen und in Deutschland Beratungstage für Versicherte und für Arbeitgeber statt. Sehr wertvoll sind aus meiner Sicht die Besprechungen der Verbindungsstellen und die Arbeitsbesprechungen, an denen Sachbearbeiter der Abteilung für Abwicklung internationaler Verträge in Oppeln und Angestellte der deutschen Versicherungsanstalten (vor allem der Deutschen Rentenversicherung Bund und der Deutschen Rentenversicherung Berlin-Brandenburg) teilnehmen. Der direkte Kontakt zwischen unseren Mitarbeitern und die Möglichkeit, sich telefonisch oder per E-Mail miteinander zur verständigen, steigert die Effizienz der Arbeit und ermöglicht eine schnelle Lösung von problematischen Angelegenheiten. 


\title{
Schaffung von grenzübergreifenden Versorgungsstrukturen in der Unfallversicherung
}

\author{
Eva-Marie Höffer
}

$\begin{array}{ll}\text { A. Einleitung } & 269\end{array}$

B. Arbeitnehmerfreizügigkeit und Dienstleistungsfreiheit 270

I. Entwicklung der Beschäftigungszahlen $\quad 271$

II. Demographie und Fachkräftemangel 272

C. Sozialversicherungsrechtliche Beurteilung von Beschäftigungen polnischer Staatsangehöriger in Deutschland 273

I. Beschäftigungslandprinzip 273

II. Entsendung 274

III. Mehrfachbeschäftigung 275

IV. Verfahren der Sachleistungsaushilfe $\quad 276$

V. Praktische Erfahrungen 276

D. Charakteristika der gesetzlichen Unfallversicherung in Deutschland 278

E. Grenzübergreifende Versorgungsstrukturen 279

F. Regelungen im Europarecht 280

I. Regelungen des koordinierenden Verordnungsrechts 281

II. Patientenrichtlinie 281

G. Anforderungen an die Umsetzung 282

H. Fazit 282

\section{A. Einleitung}

Seit dem 1. Mai 2011 gilt für polnische Staatsangehörige, die in Deutschland eine Beschäftigung aufnehmen möchten, die uneingeschränkte Arbeitnehmerfreizügigkeit.

Es stellt sich die Frage, welche sozialversicherungsrechtlichen Auswirkungen, insbesondere in Bezug auf die gesetzliche Unfallversicherung in Deutschland, hiermit verbunden sind. Dabei ist unter anderem von Interesse, ob die uneingeschränkte Arbeitnehmerfreizügigkeit zu einem Anstieg an Beschäftigungsverhältnissen in Deutschland führen wird. Konjunkturelle Entwicklungen und Trends auf dem Arbeitsmarkt können sich auf das System der gesetzlichen Unfallversicherung auswirken, das durch Unter- 